\title{
HIGH PRESSURE FREEZE-OUT OF ELECTRONS IN UNDOPED GaN CRYSTAL. PROOF OF EXISTENCE OF RESONANT DONOR STATE (NITROGEN VACANCY)
}

P. Perlin, H. Teisseyre, T. Suski, M. Leszczyński, I. Grzegory, J. Jun, AND S. POROWSKI

High Pressure Research Center "Unipress", Polish Academy of Sciences Sokołowska 29, 01-142 Warszawa, Poland

We investigated free carriers related optical absorption in $\mathrm{GaN}$ in hydrostatic pressures up to $30 \mathrm{GPa}$. The disappearance of this absorption at pressures close to $18 \mathrm{GPa}$ was explained by trapping electrons resulting from the shift of nitrogen vacancy related donor level into the GaN energy gap at high pressure. We estimated the energetic position of this level at atmospheric pressure to be about $0.8 \mathrm{eV}$ above the conduction band minimum.

PACS numbers: 71.55.-i, 71.55.Eq

\section{Introduction}

Gallium nitride is now one of the most important materials for the construction of short-wavelength light emitting devices like blue LED and lasers. In spite of impressive technological achievements of the last few years (implementation of the first commercially available blue diode) there are still many problems concerning basic physical properties of this compound that have to be solved. One of them is the question about the origin of free electrons in undoped GaN crystals.

High concentration of free electrons and serious problem in finding a suitable acceptor for this material stopped the development of GaN technology for almost two decades. Presently, $p$-type GaN has been produced by means of doping the crystal with magnesium [1], but the problem of the control of properties of an undoped material still remains. It is commonly believed that the main native defect in GaN is related to nitrogen vacancy (NV). It is suspected that this defect donates electrons to the conduction band by means of formation of the resonant donor level. It is very likely that NV appears during the process of crystal growth as a result of high nitrogen pressure at the growth temperature [2] and the reason for that is a very high equilibrium nitrogen pressure at the growth temperature. 
Although the likelihood of formation of a large number of NV was never really questioned, it was not observed experimentally. In addition, only very recently the theoretical calculations of Jenkins et al. [3] and Bogusławski et al. [4] showed that NV indeed formed a resonant donor state around $600 \mathrm{meV}$ above the $\mathrm{GaN}$ conduction band minimum. It is not easy to confirm experimentally the existence of such a resonant state, located so high in the conduction band. The most frequently used method to observe electronic resonant states is a change of the band structure by using a high pressure. In case of $\mathrm{GaN}$, this method relies on the upward shift of the bottom of the conduction band at high pressures, while the localized (and resonant) level of NV is weakly dependent upon an applied pressure. Thus at a certain pressure the deep level enters the energy gap becoming active electron trapping center. To induce an appearance of the NV related donor level in the gap of GaN one has to move the conduction band minimum by at least $600 \mathrm{meV}$. Since the energy gap widens with pressure with the rate equal to $47 \mathrm{meV} / \mathrm{GPa}$ [5] we have to apply pressure of about $14 \mathrm{GPa}$. If the deep level has some small positive pressure coefficient this crossing will occur at even higher pressure. Once the deep level falls below the conduction band minimum (or more strictly speaking below Fermi energy level) it will be populated by electrons, by means of emptying the conduction band states. It is well known that highly $n$-type GaN crystals are characterized by strong optical absorption on free carriers. This absorption for the crystals of $n=10^{19} \mathrm{~cm}^{-3}$ is observed for wavelengths higher than $1 \mu \mathrm{m}$.

\section{Experimental}

The experiment was performed in Diamond Anvil Cell (Mao-Bell like design). We applied 4:1 methanol-ethanol mixture as a pressure transmitting medium. A small piece of ruby was placed in the cell to monitor the pressure. Linear ruby scale was used to determine the pressure from the energy of ruby luminescence. The light beam from $150 \mathrm{~W}$ halogen lamp was focused on $100 \mu \mathrm{m}$ pinhole, whose image was projected on the sample. The outcoming light was dispersed by a Spex $500 \mathrm{M}$ spectrometer and detected by Peltier cooled $\mathrm{PbS}$ detector.

\section{Results}

Figure 1 represents absorption spectra of $\mathrm{GaN}$ measured for various pressures in the free carrier absorption region. For pressures lower than $15 \mathrm{GPa}$ there is no substantial change in the shape of the absorption. Between $15 \mathrm{GPa}$ and $20 \mathrm{GPa}$ the intensity of absorption decreases and for pressures higher than $20 \mathrm{GPa}$ no absorption is observed in this region. The sudden reduction of free carrier absorption observed at certain value of hydrostatic pressure provides an evidence for the presence of the localized level resonant with the conduction band (at atmospheric pressure). Assuming an independence of the level position with respect to the applied pressure makes it possible to estimate its energy to be about $0.8 \mathrm{eV}$ above the bottom of the conduction band. According to the theoretical predictions such a state could result from the formation of NV in GaN. 


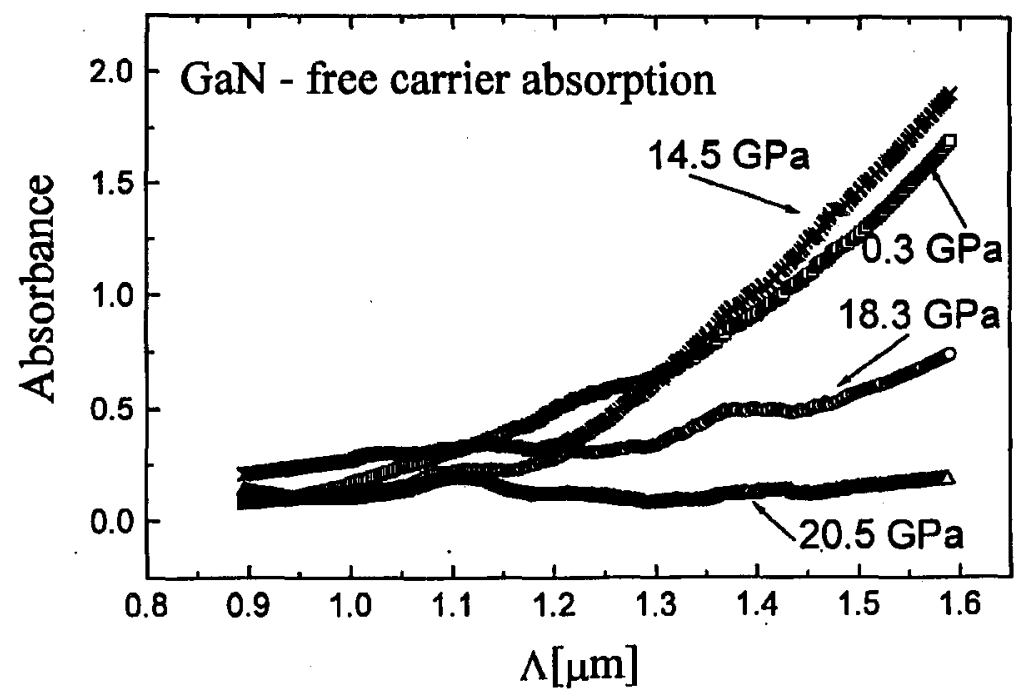

Fig. 1. Absorption spectra of $\mathrm{GaN}$ for various pressures.

\section{Acknowledgment}

Authors thank Dr. P. Mensz for valuable discussions.

\section{References}

[1] H. Amano, M. Kito, K. Hiramatsu, I. Akasaki, Jpn. J. Appl. Phys. 28, L2112 (1989). [2] I. Grzegory, S. Krukowski, Phys. Scr. Vol. T 39, 242 (1991).

[3] D. Jenkins, J.D. Dow, Ming-Hsiung Tsai, J. Appl. Phys. 72, 4130 (1992).

[4] P. Bogusławski, E. Briggs, A. White, J. Bernholc, unpublished.

[5] P. Perlin, I. Gorczyca, N.E. Christensen, P. Perlin, I. Grzegory, H. Teisseyre, T. Suski, Phys. Rev. B 45, 13307 (1992). 